\title{
Microwave cooking enhances glycaemic potential of rice: An in vitro study
}

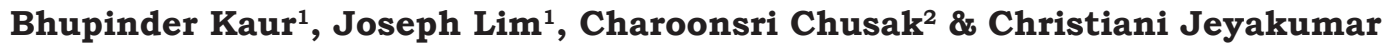 \\ Henry $^{1,3^{*}}$
}

${ }^{1}$ Clinical Nutrition Research Centre, Singapore Institute of Food and Biotechnology Innovation, Singapore; ${ }^{2}$ Department of Nutrition and Dietetics, Faculty of Allied Health Sciences, Chulalongkorn University, Bangkok, Thailand; ${ }^{3}$ National University of Singapore, Department of Biochemistry, Singapore

\begin{abstract}
Introduction: Rice is the principle staple and energy source for nearly half the world's population and has significant nutrition and health implications. Most rice varieties are considered high glycaemic index (GI) foods. With rice being a major contributor to the glycaemic load in Asian populations, there is increasing concern of its impact on the development of type 2 diabetes. Devising ways to reduce the glycaemic impact of rice is therefore imperative. Rice is cooked in a variety of ways, such as over an open fire, electric cooker or microwave. We evaluated the impact of the electric cooker and microwave cooking methods on starch digestibility in a variety of rice commonly consumed in Asia. Methods: Nine rice varieties of varying GIs were cooked in an electric cooker and microwave oven, and the starch digestibility of cooked rice measured using an in vitro digestion method. Results: High GI white rice (WR) had reduced starch digestibility after microwave cooking compared to electric cooker $(p<0.05)$. There was a significant reduction in incremental area under the curves (iAUC) and rapidly digestible starch of WR cooked using microwave rather than electric cooker $(p<0.05)$. Interestingly, even for low GI rice varieties such as iddly rice and extra long basmati rice, microwaving increased slowly digestible starch. Conclusion: The results provide indicative data that the glycaemic impact of rice can be reduced when cooked using a microwave oven. These observations have nutritional implications that need to be substantiated using in vivo studies. Microwaving may provide a simple method of reducing the postprandial glycaemia of high GI rice.
\end{abstract}

Keywords: Cooking method, microwave cooking, rice, starch digestibility, glycaemic index

\section{INTRODUCTION}

Rice (Oryza sativa) is an important staple food of an estimated 3.5 billion people worldwide, with more than $90 \%$ of rice being consumed in Asia (Mohanty, 2013).
The major component of rice is starch, which provides substantial energy for human needs. It also has a high protein digestibility, biological value and protein efficiency ratio owing to the greater concentration of lysine among all the

\footnotetext{
*Corresponding author: Prof. Christiani Jeyakumar Henry

Clinical Nutrition Research Centre, Singapore Institute of Food and Biotechnology Innovation, 14 Medical Drive \#07-02, Singapore 117599, Singapore.

Tel: (65) 6407 0793; Fax: (65) 6776 6840; E-mail: jeya_henry@sifbi.a-star.edu.sg doi: https://doi.org/10.31246/mjn-2019-0106
} 
cereals (Eggum, 1979). The glycaemic index (GI) and glycaemic load (GL) are measures used to estimate and rank the postprandial glycaemic response to carbohydrate-containing foods. More specifically, the GI is a simple way of characterising carbohydrates based on their impact on postprandial glycaemic response (Jenkins et al., 1981a). Carbohydrates with a low GI value $(<55)$ are more slowly digested, absorbed and metabolised, and lead to a slower rise in blood glucose than those higher in GI values (Jenkins et al., 1981b). Using GI, GL can be calculated by multiplying the food's specific GI with the consumed quantity of carbohydrate in that food. Recent epidemiological studies suggest that rice is potentially a significant contributor to the diabetes incidence, especially in Asian communities where rice is a major contributor to $\mathrm{GL}(\mathrm{Hu}$ et al., 2012). In several studies, rice has been reported to be a high GI food (>70) (Jenkins et al., 1981a, Miller, Pang \& Bramall, 1992). The rising rates of diabetes in Asia coupled with the excessive consumption of high GI rice means that there is a need to find ways to reduce the glycaemic impact of rice (Zheng, Ley \& Hu, 2018). While there are many processed rice products in the market today (Juliano, 1993), rice is primarily consumed in the form of cooked grains. A recent extensive review has shown that one of the major factors affecting digestibility of rice is the method of cooking (Kaur, Ranawana \& Henry, 2016). During cooking, changes occur in the rice structure, which in turn alter the nutritional characteristics, including starch digestibility. Some studies have also reported that cooking methods can affect the starch digestibility of rice. The nutritional composition of rice varieties may be influenced by cooking methods depending upon techniques and conditions including temperature, time and moisture (Han, Lee \& Rhee, 2008; Reed et al., 2013). For centuries, rice has been cooked over an open flame (or other sources of heat) with water, by a rapid/ gentle boil method (Bhattacharya, 2011). The development of the electric rice cooker automated the process of cooking rice by mechanically or electronically controlling heat and timing. The electric rice cooker was invented and was first introduced by Toshiba Japan in 1955. With the first introduction and the availability of power grids to domestic users, the use of electric cooker became widespread (Ito, 2017). With an electric rice cooker, the user's involvement in cooking rice is reduced to simply measuring the rice, preparing the rice properly and using the correct amount of water. Once the rice cooker is set to cook, no further attention will be needed during the cooking duration. However, unlike an electric rice cooker that does not necessarily speed up the cooking process (cooking time of up to $30 \mathrm{~min}$ ), microwave ovens do reduce cooking time (cooking time of $\leq 15 \mathrm{~min}$ ). In recent years, there has been an increased ownership of domestic microwave ovens, which has revolutionised the way we cook rice. In microwave cooking, foods are cooked by frictional heat produced by the action of microwaves on water molecules, causing them to vibrate at high speed (Khatoon \& Prakash, 2006).

The present study is to investigate the effect of cooking methods on starch digestibility of rice. Our aim was to investigate the glycaemic impact of rice cooked using an electric cooker and a microwave oven, assessed using an in vitro starch digestion method. In addition to the commonly consumed white rice, we also compared eight other rice varieties with different GIs, chosen based on their popularity in Asia. 


\section{MATERIALS AND METHODS}

\section{Rice samples}

All rice samples were purchased in Singapore. The nine rice varieties chosen were Thai Hom Mali Jasmine white rice (WR), premium Calrose rice (CR), Kangaroo Australian low GI rice (LGR), iddly rice (IR), fresh brown Jasmine rice (BJR), extra long Basmati rice (LBR), premium Thai red rice (TRR), white glutinous rice (WGR) and black glutinous rice (BGR). The proximate nutrient composition and GI of these rice samples are provided in Table 2 . The GI of rice samples were also obtained from our previous work and from the literature (Table 2). The rice variety, abbreviation, country of known origin, pre-cooking preparation and cooking conditions are presented in Table 1.

\section{Chemicals and reagents}

Alpha-amylase from porcine pancreas type VI-B, pepsin from porcine gastric mucosa powder, maleic acid, sodium hydroxide $(\mathrm{NaOH})$, bile extracts porcine, amyloglucosidase from Aspergillus niger, pancreatin from porcine pancreas, sodium acetate trihydrate, 3,5-dinitrosalicylic acid and potassium sodium tartrate were purchased from Sigma-Aldrich (MO, USA). Hydrochloric acid $(\mathrm{HCl})$, ethanol and acetic acid were obtained from Merck (Darmstadt, Germany). Glucose was purchased from Kento Chemical Co., Inc. (Tokyo, Japan).

\section{Preparation and cooking of rice samples}

The nine rice samples were cooked using an electric rice cooker and a microwave oven (R-398F, SHARP CORP, Japan). For each cooking method and rice variety, the optimum time and procedures were established before the actual experimental testing (Table 2). Rice were cooked until no white core remained in the rice kernels (Lum, 2017).
For electric rice cooking, WR, CR, LGR, IR and LBR were washed and drained thrice with water and then placed into the electric rice cooker with a rice:water ratio of $1: 1.4$ [weight/volume $(\mathrm{w} / \mathrm{v})]$. A rice water ratio of $1: 2(\mathrm{w} / \mathrm{v})$ was used for BJR or TRR. WGR and BGR were soaked for $30 \mathrm{~min}$ and placed into the rice cooker by rice:water ratio of $1: 1.4(\mathrm{w} / \mathrm{v})$ and $1: 2(\mathrm{w} / \mathrm{v})$, respectively. Rice was wrapped in aluminium foils to further keep warm until ready for digestion. Digestion of samples began within 20 min after cooking.

For microwave cooking, a rice:water ratio of $1: 1.8(\mathrm{w} / \mathrm{v})$ was used for the cooking of WR, CR, LGR, IR, LBR and WGR. Prior to microwaving, these rice samples were washed and drained thrice with water. BJR, TRR and BGR were cooked with a rice:water ratio of $1: 2.5$ (w/v) (Table 1). The cooked rice was left at room temperature $\left(25^{\circ} \mathrm{C}\right)$ until analysis. Rice was wrapped in aluminium foils to further keep warm until ready for digestion. Digestion of samples began within 20 min after cooking.

\section{In vitro digestion of rice}

The glycaemic potential of the cooked rice samples were assessed using a pre-validated in vitro starch digestibility model, that mimics the human gastrointestinal digestion (Mishra \& Monro, 2009, Monro et al., 2010). This method has been shown to provide accurate, indicative data on the in vivo glycaemic response.

Rice samples $(2.5 \mathrm{~g})$ with $30 \mathrm{~mL}$ of distilled water was digested in specimen pots and inserted into a 12-position aluminum block placed in a circulating water bath maintained at $37^{\circ} \mathrm{C}$ with stirring at $130 \mathrm{rpm}$. After stirring for $30 \mathrm{~min}$, the oral phase was initiated by the addition of $100 \mu \mathrm{L}$ of $10 \% \alpha$-amylase ( $\geq 10$ units $/ \mathrm{mg}$ solid) in distilled water for $1 \mathrm{~min}$. Then, $200 \mu \mathrm{L}$ of $1 \mathrm{M} \mathrm{HCL}$ was added to the samples in quick succession 
Kaur B, Lim J, Chusak C et al.

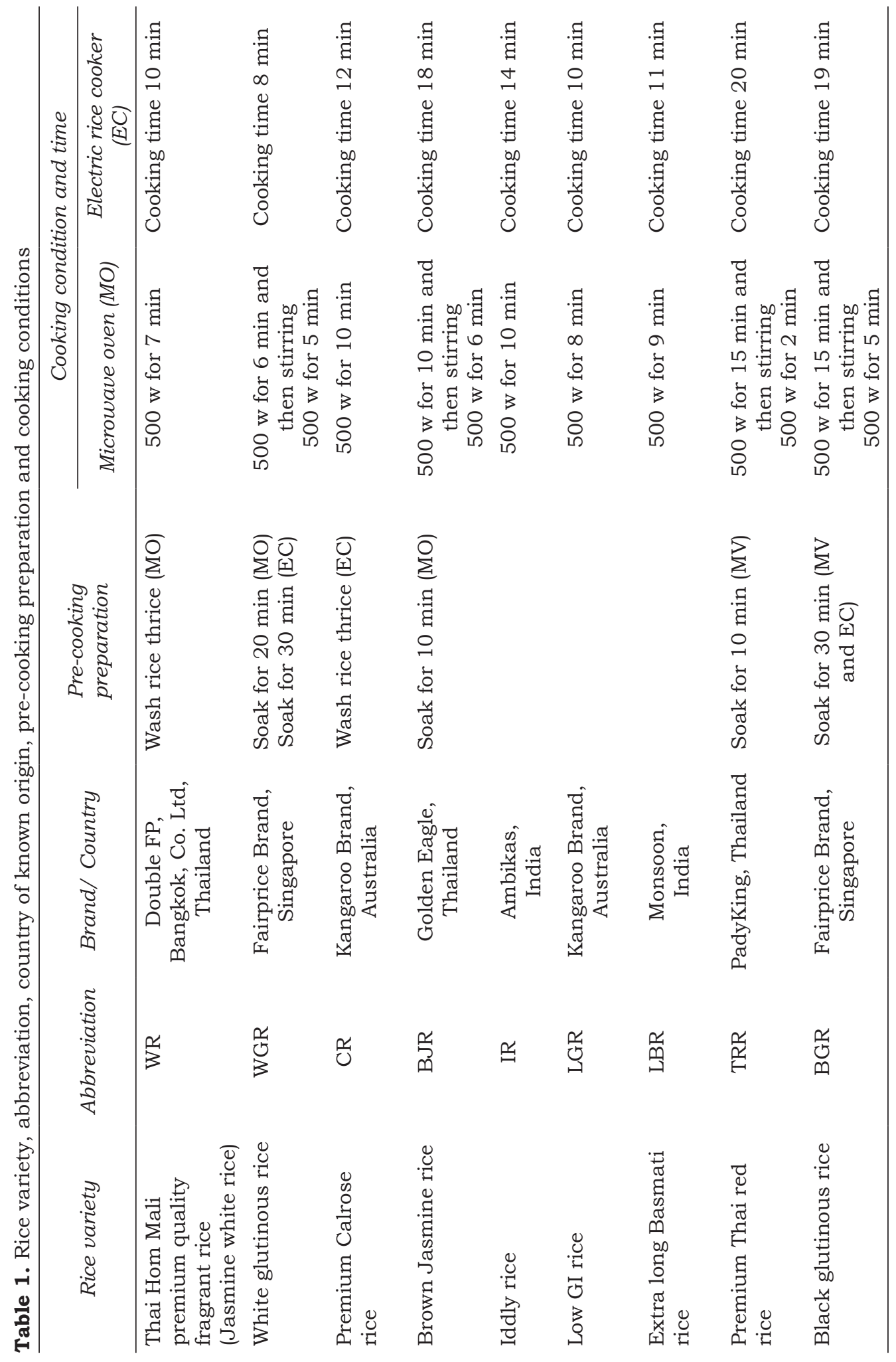


Table 2. Proximate composition of rice samples

\begin{tabular}{lccccc}
\hline \multirow{2}{*}{\multicolumn{1}{c}{ Types of rice }} & \multirow{2}{*}{$\begin{array}{c}\text { Glycaemic } \\
\text { index (GI) }\end{array}$} & $\begin{array}{c}\text { Total } \\
\text { carbohydrate }\end{array}$ & $\begin{array}{c}\text { Available } \\
\text { carbohydrate }\end{array}$ & $\begin{array}{c}\text { Total } \\
\text { protein }\end{array}$ & $\begin{array}{c}\text { Total } \\
\text { fat }\end{array}$ \\
\hline Thai Hom Mali Jasmine white & 91 & 81.26 & 76.55 & 6.94 & 0.38 \\
rice (WR) & & & & & \\
White glutinous rice (WGR) & 93 & 81.80 & 76.85 & 7.25 & 0.18 \\
Premium Calrose rice (CR) & 83 & 78.79 & 73.67 & 7.46 & 0.80 \\
Brown Jasmine rice (BJR) & 74 & 76.59 & 68.59 & 7.80 & 2.91 \\
Iddly rice (IR) & 38 & 81.16 & 73.96 & 8.05 & 0.57 \\
Low GI rice (LGR) & 54 & 80.60 & 70.72 & 7.54 & 0.65 \\
Extra long Basmati rice (LBR) & 52 & 78.96 & 71.04 & 9.18 & 1.27 \\
Premium Thai red rice (TRR) & 55 & 75.78 & 65.64 & 8.62 & 2.66 \\
Black glutinous rice (BGR) & 42 & 72.86 & 66.84 & 10.71 & 2.09 \\
\hline
\end{tabular}

to adjust it to $\mathrm{pH} 2.5( \pm 0.2)$, measured by the $\mathrm{pH}$ meter (SevenCompact ${ }^{\mathrm{TM}}$, METTLER TOLEDO ${ }^{\circledR}$, OH, USA). Gastric phase was then initiated by the addition of $1 \mathrm{~mL}$ of $10 \%$ pepsin ( $\geq 250$ units / mg solid) dissolved in $0.05 \mathrm{M} \mathrm{HCl}$. The mixture was continuously stirred at $37^{\circ} \mathrm{C}$ for $30 \mathrm{~min}$ to complete the gastric digestion phase. After which, $2 \mathrm{~mL}$ of 1 $\mathrm{M} \mathrm{NaHCO}$ and $5 \mathrm{~mL}$ of $0.2 \mathrm{M}$ maleate buffer ( $\mathrm{pH} \mathrm{6)}$ were added into the mixture to neutralise gastric $\mathrm{HCl}$. Five millilitres of $10 \%$ bile extract solution in distilled water was added to the mixture and then distilled water was filled into the pots to adjust the volume to $55 \mathrm{~mL}$. After holding for $15 \mathrm{~min}$ to reach $37^{\circ} \mathrm{C}$, the pancreatic phase was started by adding $100 \mu \mathrm{L}$ of amyloglucosidase $(\geq 260 \mathrm{U} / \mathrm{mL}$, aqueous solution) and $1 \mathrm{~mL}$ of $5 \%$ pancreatin in $0.2 \mathrm{M}$ maleate buffer. An aliquot of $250 \mu \mathrm{L}$ from baseline, the end of oral and gastric phase, and at 20, 60, 90, 120 and 180 min from the start of pancreatic phase was drawn and transferred into tubes containing $1 \mathrm{~mL}$ of ethanol to end the enzymatic digestion (Monro et al., 2010, Mishra \& Monro, 2009).

\section{Analysis of reducing sugars released during in vitro digestion}

Reducing sugars released from the rice samples during in vitro digestion (ethanolic digesta) was measured by Dinitrosalicylic acid (DNS) colourimetric method (Englyst \& Hudson, 1987). The ethanolic digesta samples were centrifuged at $1000 \mathrm{rpm}$ for $10 \mathrm{~min}$. Fifty microlitres of aliquots from the supernatant were removed and mixed with $250 \mu \mathrm{L}$ of $0.1 \mathrm{M}$ acetate buffer $(\mathrm{pH}$ 5.2) containing $1 \%$ amyloglucosidase ( $\geq 260 \mathrm{U} / \mathrm{mL}$, aqueous solution). After incubation at $37^{\circ} \mathrm{C}$ for $10 \mathrm{~min}, 750 \mu \mathrm{L}$ of DNS mixture $10.5 \mathrm{mg} / \mathrm{mL}$ of glucose: $4 \mathrm{M} \mathrm{NaOH}$ :DNS reagent mixed in ratio at $1: 1: 5)$ was added. The mixture was heated at $95-100^{\circ} \mathrm{C}$ for $15 \mathrm{~min}$ and then $4 \mathrm{~mL}$ of distilled water was added to each sample. After cooling at room temperature, absorbance was read at $530 \mathrm{~nm}$ by a microplate reader (Infinite ${ }^{\circledR} 200$ PRO, Tecan Trading AG, Switzerland). Glucose (10mg/mL) and distilled water were used as standard and blank, respectively. The results were expressed as $\mathrm{mg} / \mathrm{mL}$ of glucose. 


\section{Determination of rapidly digestible starch, slowly digestible starch and undigested starch}

Rapidly digestible starch (RDS) was calculated as the amount of digested starch within the initial 20 min (G20) after the start of pancreatic phase (Englyst et al., 1999). Slowly digestible starch (SDS) was defined as the amount of digested starch between 20 and 120 min (G120). Undigested starch (US) was considered as the amount that was not digested within $120 \mathrm{~min}$. Each fraction was calculated as follows: $\mathrm{RDS}=(\mathrm{G} 20$ Free Glucose ${ }^{*} 0.9$; $\mathrm{SDS}=(\mathrm{G} 120-\mathrm{G} 20)^{*} 0.9$; $\mathrm{US}=(\mathrm{G} 180-120) * 0.9$. The conversion factor is 0.9 from glucose to starch (Englyst et al., 1999).

\section{Statistical analysis}

All experiments were carried out in triplicate. The data were presented as mean \pm standard error of mean (SEM). The comparisons between samples at each individual time point were analysed by one-way analysis of variance (ANOVA) with Duncan's multiple comparison test (SPSS version 17; SPSS Inc., IL, USA). Differences in the incremental area under the glucose curves (iAUC) and amount of RDS and SDS between the samples were determined by one-way ANOVA with Duncan's multiple comparison test. In addition, independent $t$-test was used to analyse iAUC and amounts of RDS, SDS and US for electric cooked and microwave cooked rice samples. Statistical significance was set at $p<0.05$.

\section{RESULTS}

The starch digestion curves of rice cooked by an electric cooker (A) and microwave oven (B) are illustrated in Figure 1. WR cooked in an electric cooker had a significantly higher rate of glucose release compared to microwave cooking for all phases of the in vitro model $(p<0.05)$. For electric cooking, WR had the highest amount of incremental glucose concentration during the time of digestion. The incremental glucose concentrations of WR were significantly higher at oral phase and at 60, 90, 120 and $180 \mathrm{~min}$ of intestinal phase compared to all the other rice varieties $(p<0.05)$. Figure 2 displays the iAUC for glucose release of all rice varieties for both cooking methods. The iAUC for WR cooked using electric cooker was 1.4 times higher than microwave cooking $(p<0.05)$. Although there were no significant differences for the other rice varieties, it was noteworthy that microwaving high GI varieties such as CR, WGR and BJR showed reduction in iAUCs (Figure 2). Furthermore, low GI rice varieties, with the exception of IR, also saw reductions in iAUC when microwave cooking was applied (Figure $2)$. WR cooked in a microwave oven showed a significant decrease in RDS compared to cooking in an electric cooker $(23.5 \mathrm{~g}$ vs $34.6 \mathrm{~g} / 100 \mathrm{~g})(p<0.05)$ (Figure 3). There was an overall trend showing higher RDS values for high GI rice varieties (except BJR) compared to the low GI rice varieties, irrespective of cooking method (Figure 3). BJR showed the highest quantity of undigested starch when microwaved $(p<0.05)$ (Figure 3). Both BGR and TRR had no differences in quantities for each starch fraction (RDS, SDS, US), irrespective of cooking methods. Both LBR and IR had significantly higher quantities of SDS when microwaved compared to electric cooking (LBR: 28.8 vs $16.4 \mathrm{~g} / 100 \mathrm{~g}$ and IR: $26.8 \mathrm{~g}$ vs $15.3 \mathrm{~g} / 100 \mathrm{~g})(p<0.05)$.

\section{DISCUSSION}

Several studies have investigated the digestibility of rice cooked using various cooking methods (Khatoon et al., 2006; Lee et al., 2005; Li et al., 2014; Rashmi \& Urooj, 2003; Chiu \& Stewart, 2013). To our knowledge, this is the first to 

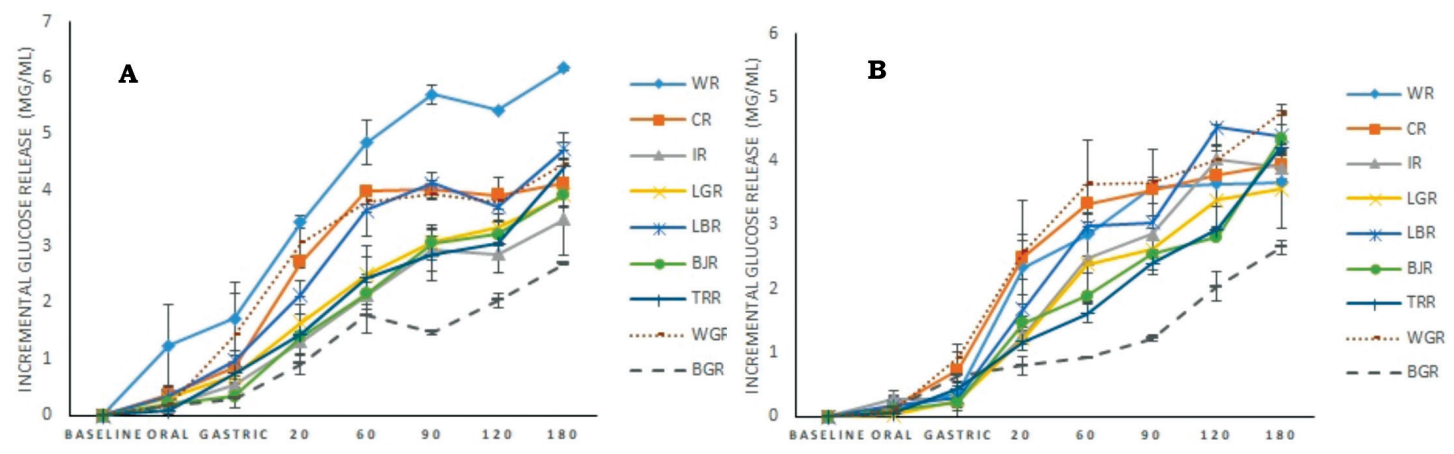

Figure 1. The incremental glucose released from rice varieties cooked using (A) an electric cooker and (B) microwave oven during in vitro digestibility. Results expressed as mean $\pm S E M$

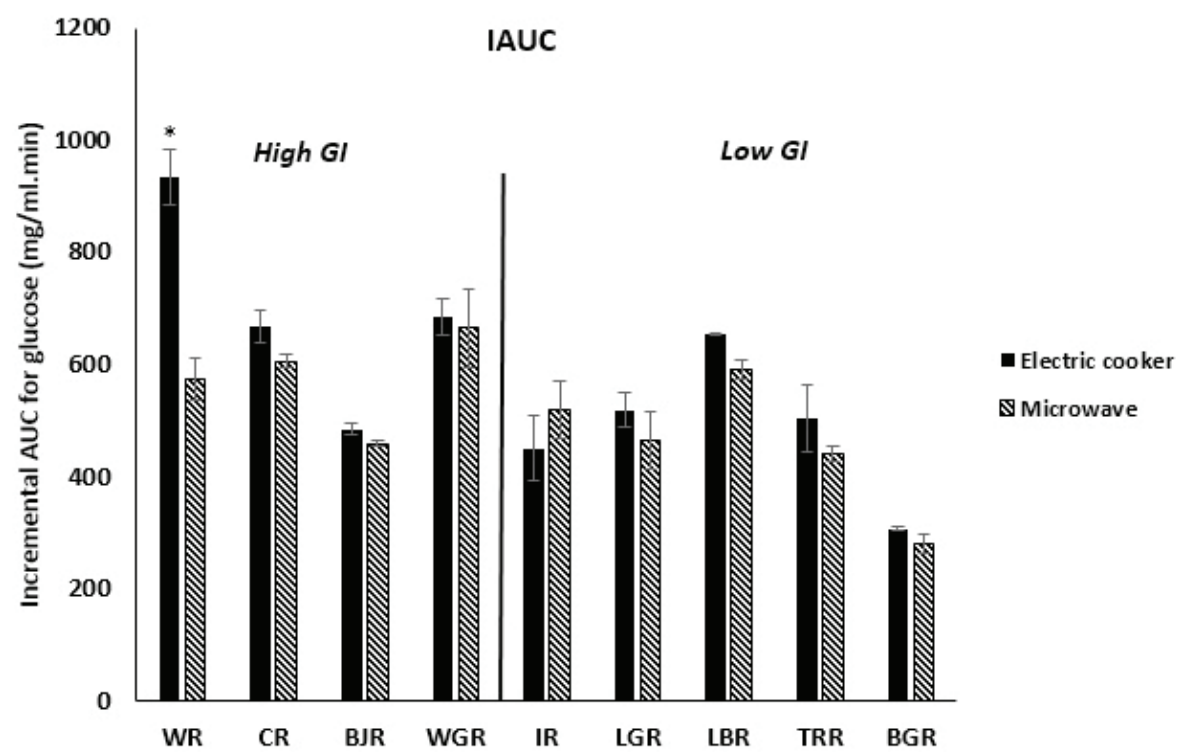

Figure 2. Incremental area under the curve (iAUC) for glucose release of rice varieties cooked using an electric cooker and a microwave oven. Results expressed as mean $\pm S E M$

*Mean values were significantly different between electric cooker and microwave cooking $(p<0.05)$

examine starch digestibility of a range of low and high GI rice consumed in this part of Asia, which were cooked using both an electric cooker and a microwave oven.

\section{Starch digestibility}

WR cooked in a microwave oven had a distinctly slower rate of glucose release compared to electric rice cooking. There was also a small reduction in starch digestibility of other rice varieties (except for IR) when microwaving was applied compared to electric cooking. Both methods of cooking had a cooking temperature of $100^{\circ} \mathrm{C}$, however, the time to reach the boiling temperature was much shorter for microwave cooking (4 min and 41 seconds), versus electric cooking (5 min 22 seconds). The disrupted starch 

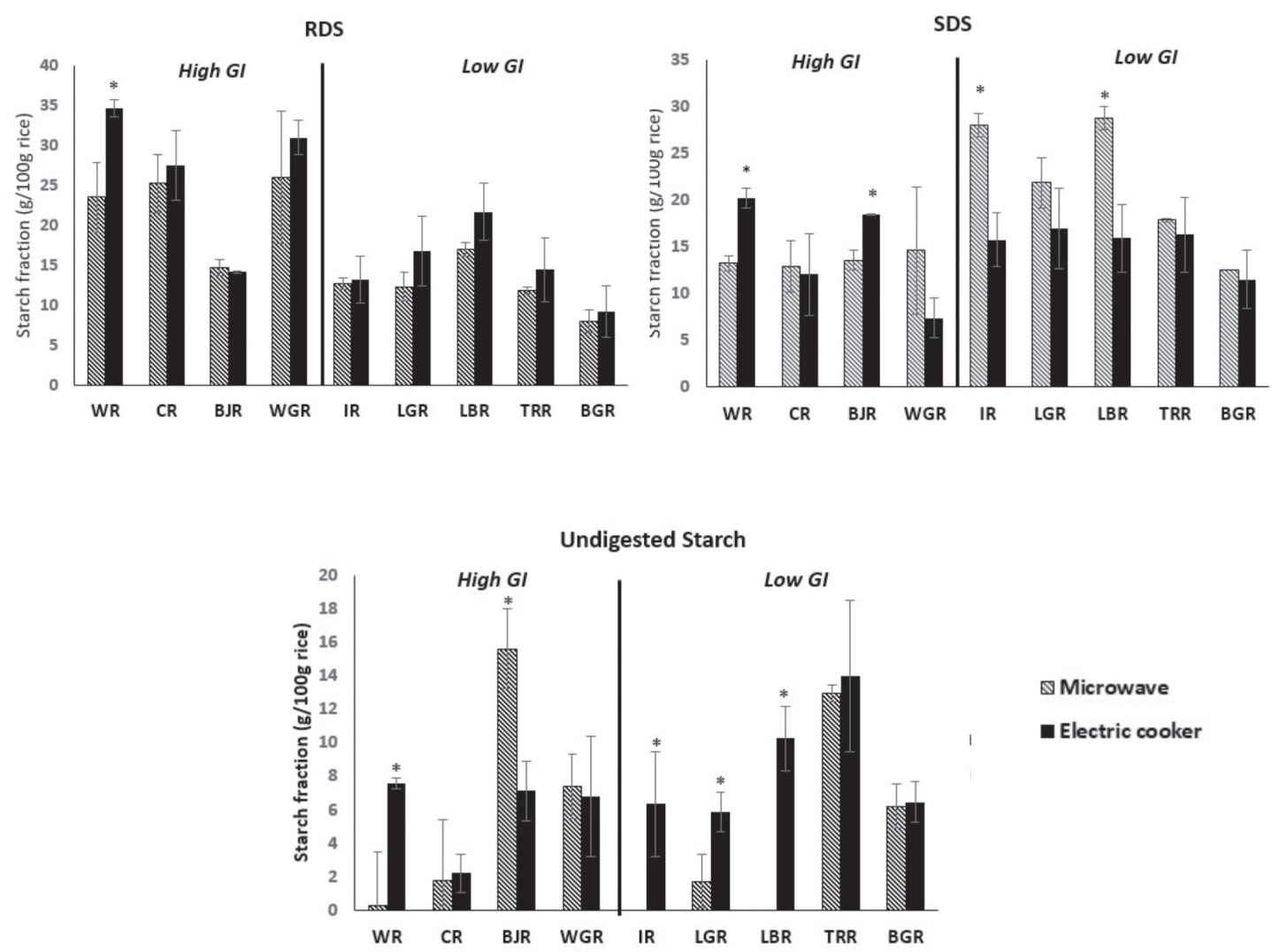

Figure 3. RDS, SDS and US of rice varieties cooked by electric cooker and microwave oven. Results expressed as mean $\pm S E M$

* Mean values were significantly different between electric cooker and microwave cooking ( $p<$ 0.05)

structure during gelatinisation makes it more accessible to digestive enzymatic degradation and the glycaemic impact of the starch rises (Holm et al., 1988). Microwave cooking has been reported to generate less dispersed gelatinised starch than conventional cooking of starch products, thereby altering the digestibility of starch (Yiu, Weisz \& Wood, 1991; Sánchez-Pardo et al., 2007). It has also been shown that the chemical bonds, chemical groups, skeleton, and the way that rice starch are connected with each other are not destroyed by microwave treatment as compared to conventional heating methods (Fan et al., 2012). Moreover, the shorter cooking time involved with microwaving, results in a reduction in the degree of starch gelatinisation. Another point to note was that the rice to water ratio required for cooking rice using the electric cooker was higher than the microwave oven, which could contribute to increased starch gelatinisation (with the exception of BJR, TRR and BGR which required more water to be completely cooked with microwave oven cooking). This finding is one that is very important, especially for the cooking of white rice, which is a high GI staple commonly consumed in Asia. Microwaving white rice may reduce its glycaemic impact. 


\section{RDS, SDS and US}

It is well recognised that starch can be classified based on its rate of glucose release and its absorption in the gastrointestinal tract, where they can be classified into RDS, SDS and US (Englyst, Kingman \& Cummings, 1992). The RDS content in food is a reflection of the amount of readily digestible starch that is largely accountable for the shortterm postprandial glycaemic response, whilst SDS represents the slowly digested starch fraction that produces a low and protracted glycaemic response (Englyst et al., 1992; Englyst et al., 1999). US, or resistant starch, is not digested by carbohydrate digestive enzymes in the small intestine. Cooking WR using the electric cooker produced higher RDS and increased US, which was also observed in a study by Chung and team, where this finding was attributed to the amylose content of white rice (Chung et al., 2010). Microwave cooking had the ability to reduce RDS in WR and slow down the rate of digestibility. This has a beneficial role in reducing the glycaemic impact of high RDS-containing foods such as white rice by producing a slower release of glucose. There was an overall trend of generally higher RDS values for the high GI rice varieties (except BJR) compared to the low GI rice varieties, irrespective of cooking methods. Using an electric cooker, BJR had the longest cooking time compared to other high GI rice varieties. The cooking time primarily determines the extent of starch gelatinisation and as a consequence, the digestibility of rice (Ranawana et al., 2009). Interestingly, BJR produced the largest amount of US (resistant starch) when cooked in the microwave oven. Although BJR is a high GI rice (lower amylose content), with a GI of 74 , the starch present in BJR may not be as susceptible to enzymatic hydrolysis as compared to the starch present in WR, WGR and CR. A review by Touttounji et al. (2019) pointed out that even though the starch digestibility of rice is primarily attributed to the amylose content, but the amylopectin fine structure can also play a contributory role. The long chain amylopectin which mimics the structure, and therefore the function of amylose, can also increase resistant starch and reduce the digestibility of rice (Butardo et al., 2017). The increased formation of undigested starch with BJR after microwave cooking could possibly be due to the formation of resistant starch after the cooking process (resistant starch type III), formed in part due to the retrogradation of amylose (Guillén, Oria \& Salvador, 2018) and the presence of polyphenols in BJR that could bind with starch molecules (Panlasigui \& Thompson, 2006). Rice cooked in a microwave oven has been reported to have a lower RDS content than autoclaved and parboiled rice (Niba, 2003). Microwave processed starches have been shown to have a higher SDS level and a lower digestion rate, which was also observed in our study (Niba, 2003). This in vitro observation has potential nutritional implications especially for white rice, which is a very high GI food that causes sharp rises in blood glucose. Both BGR and TRR had no differences in their quantities for each starch fraction (RDS, SDS, US), irrespective of cooking methods. The presence of dietary fibre, bran, anthocyanins and other phytochemicals act as inhibitors to enzymatic digestion. The dietary fibre-rich bran fraction can act as a barrier to digestive enzyme activity, thereby reducing starch digestibility (Tian, Nakamura \& Kayahara, 2004, Panlasigui \& Thompson, 2006). There was an increase in SDS for LBR when microwaved compared to electrically cooked. An in vivo study on LBR also showed a reduction in glycaemic response when cooked in a microwave compared to cooking in a rice cooker 
(Gunathilaka \& Ekanayake, 2015). IR, which is a parboiled rice, is considered to be a slow digesting product and from our findings, it had the highest SDS quantity with microwaving compared to electric cooking. There was also no US remaining with microwaving IR, which makes it appear to be also a very digestible food. This is possibly due to parboiled rice being relatively low in amylose $(13.1 \%)$ and being already semi-cooked (soaked, steamed and dried during parboiling operation) (Dhital et al., 2015). Parboiled rice has also been shown to have a very low pasting property due to its granules already being gelatinised (amylose leached out) during the parboiling process and there is no further swelling of the starch granules (Hermansson \& Svegmark, 1996).

\section{Comparing low and high GI cooked rice varieties}

All high GI and low GI rice varieties (except IR), had reduced iAUCs when microwave cooking was applied compared to the electric cooker. Although this reduction in iAUC was not significant, except for WR, this pattern of reduced iAUCs may have important nutritional implications. In addition, high proportions of SDS and lower RDS values were observed with the low GI rice varieties when microwave cooking was applied compared to electric cooking, but this trend was not observed with the high GI rice varieties. The application of microwave cooking indeed influenced the nutritional quality of low and high GI rice varieties, even though a significant difference was observed only with white rice. Previous studies on rice starch digestibility led to the conclusion that rice should generally be classified as a high GI/glycaemic response food (Miller et al., 1992, Jenkins et al., 1981a). In contrast, a study by Frei, Siddhuraju \& Becker (2003) revealed that the selection of a certain rice cultivar (low and high GI rice varieties), as well as the adaptation of processing/cooking might offer the possibility of substantially reducing the glycaemic response of ingested rice (Frei et al., 2003). Another in vivo study reported a reduction in GI of two Pakistani basmati rice varieties when microwave oven was used compared to an electric cooker (Gunathilaka \& Ekanayake, 2015). Our study observed an increase in SDS which could possibly indicate a reduction in GI when microwave cooking was applied. Future investigations could look deeper into the texture qualities of cooked rice, degree of gelatinisation and amylose-amylopectin structure of these rice varieties. A comparison of the changes occurring before and after cooking, with these two methods, would be an important area to explore.

\section{CONCLUSION}

The present study reports that starch digestibility can be significantly altered when rice is cooked using a microwave oven compared to an electric cooker. Microwave cooking could be recommended as a simple tool to lower the glycaemic response of high GI rice, such as white rice. In many countries in Asia, where large quantities of rice are consumed, microwave cooking is a simple and practical way to reduce the glycaemic response of rice. Given the widespread interest in consuming low GI foods in Asia, the potential intervention of using simple culinary methods to reduce glycaemic response maybe through (a) choosing appropriate cooking methods such as microwaving (b) selecting low GI rice varieties, (c) selecting black, brown or purple rice over white rice. However, further research is necessary to translate in vitro observations into in vivo human trials to validate these findings. 


\section{Acknowledgement}

This study was supported by the Biomedical Science Institute Industry Alignment Fund (IAFPP), Food Structure Engineering for Nutrition and Health (H17/01/a0/A11 \& H18/01/a0/B11).

\section{Authors' contributions}

BK, conducted data analysis and interpretation, drafted and reviewed manuscript; JL, conducted data analysis and interpretation, drafted and reviewed manuscript; $\mathrm{CC}$, carried out in-vitro work and analysed the data; $\mathrm{CJH}$, conducted data analysis and interpretation, drafted and reviewed manuscript.

\section{Conflict of interest}

All authors declare no conflict of interest.

\section{Reference}

Bhattacharya KR (2011). Rice quality: A guide to rice properties and analysis, Elsevier.

Butardo VM, Anacleto R, Parween S, Samson I, De Guzman K, Alhambra CM, Misra G \& Sreenivasulu N (2017). Systems genetics identifies a novel regulatory domain of amylose synthesis. Plant Physiol 173:887-906.

Chiu YT \& Stewart ML (2013). Effect of variety and cooking method on resistant starch content of white rice and subsequent postprandial glucose response and appetite in humans. Asia Pac J Clin Nutr 22:372-9.

Chung HJ, Liu Q, Huang R, Yin Y \& Li A (2010). Physicochemical properties and in vitro starch digestibility of cooked rice from commercially available cultivars in Canada. Cereal Chem 87:297-304.

Dhital S, Dabit L, Zhang B, Flanagan B \& Shrestha AK (2015). In vitro digestibility and physicochemical properties of milled rice. Food Chem 172:757-765.

Eggum B (1979). The nutritional value of rice in comparison with other cereals. Proceedings of Workshop on Chemical Aspects of Rice Grain Quality, 91-111.

Englyst HN \& Hudson GJ (1987). Colorimetric method for routine measurement of dietary fibre as non-starch polysaccharides. A comparison with gas-liquid chromatography. Food Chem 24:63-76.

Englyst HN, Kingman S \& Cummings J (1992). Classification and measurement of nutritionally important starch fractions. Eur $J$ Clin Nutr 46:S33-50.
Englyst KN, Englyst HN, Hudson GJ, Cole TJ \& Cummings JH (1999). Rapidly available glucose in foods: an in vitro measurement that reflects the glycemic response. Am J Clin Nutr 69:448-454.

Fan D, Ma W, Wang L, Huang J, Zhao J, Zhang $\mathrm{H} \&$ Chen W (2012). Determination of structural changes in microwaved rice starch using Fourier transform infrared and Raman spectroscopy. Starch-Stärke 64:598-606.

Frei M, Siddhuraju P \& Becker K (2003). Studies on the in vitro starch digestibility and the glycemic index of six different indigenous rice cultivars from the Philippines. Food Chem 83:395-402.

Guillén S, Oria R \& Salvador ML (2018). Impact of cooking temperature on in vitro starch digestibility of rice varieties with different amylose contents. Pol J Food Nutr Sci 68:319325.

Gunathilaka M \& Ekanayake S (2015). Effect of different cooking methods on glycaemic index of Indian and Pakistani basmati rice varieties. Ceylon Med J 60:57-61.

Han SH, Lee SW \& Rhee C (2008). Effects of cooking methods on starch hydrolysis kinetics and digestion-resistant fractions of rice and soybean. Eur Food Res Technol 227:1315-1321.

Hermansson AM \& Svegmark K (1996). Developments in the understanding of starch functionality. Trends Food Sci Technol 7:345353.

Holm J, Lundquist I, Björck I, Eliasson AC \& Asp NG (1988). Degree of starch gelatinization, digestion rate of starch in vitro, and metabolic response in rats. Am J Clin Nutr 47:1010-1016.

Hu EA, Pan A, Malik V \& Sun Q (2012). White rice consumption and risk of type 2 diabetes: meta-analysis and systematic review. $B M J$ 344:e1454.

Ito M (2017). The circular evolution and history of Japanese rice cookers [Online]. Japan: The Japan Times, Ltd. From https://www. japantimes.co.jp/life/2017/07/22/food/ circular-evolution-history-japanese-ricecookers/\#.Xi_7IGgzbIV [Retrieved April 23 2020].

Jenkins DJ, Wolever T, Taylor RH, Barker H, Fielden H, Baldwin JM, Bowling AC, Newman HC, Jenkins AL \& Goff DV (1981a). Glycemic index of foods: a physiological basis for carbohydrate exchange. Am J Clin Nutr 34:362366. 
Jenkins DJ, Wolever TM, Taylor RH, Barker $\mathrm{H}$, Fielden H, Baldwin JM, Bowling AC, Newman HC, Jenkins AL \& Goff DV (1981b). Glycemic index of foods: a physiological basis for carbohydrate exchange. Am J Clin Nutr 34:362-6.

Juliano BO (1993). Rice in Human Nutrition, International Rice Research Institute. Manila.

Kaur B, Ranawana V \& Henry J (2016). The glycemic index of rice and rice products: a review, and table of GI values. Crit Rev Food Sci Nutr 56:215-236.

Khatoon N \& Prakash J (2006). Nutritional quality of microwave and pressure cooked rice (Oryza sativa) varieties. Food Sci Technol Int 12:297305.

Lee SW, Lee JH, Han SH, Lee JW \& Rhee C (2005). Effect of various processing methods on the physical properties of cooked rice and on in vitro starch hydrolysis and blood glucose response in rats. Starch-Stärke 57:531-539.

Li J, Han W, Xu J, Xiong S \& Zhao S (2014). Comparison of morphological changes and in vitro starch digestibility of rice cooked by microwave and conductive heating. StarchStärke 66:549-557.

Lum MS (2017). Physicochemical characteristics of different rice varieties found in Sabah, Malaysia. Transactions on Science and Technology 4:68-75.

Miller JB, Pang E \& Bramall L (1992). Rice: a high or low glycemic index food? Am $\mathrm{J}$ Clin Nutr 56:1034-1036.

Mishra S \& Monro JA (2009). Digestibility of starch fractions in wholegrain rolled oats. J Cereal Sci 50:61-66.

Mohanty S (2013). Trends in global rice consumption. Rice Today 12:44-45.

Monro JA, Wallace A, Mishra S, Eady S, Willis JA, Scott RS \& Hedderley D (2010). Relative glycaemic impact of customarily consumed portions of eighty-three foods measured by digesting in vitro and adjusting for food mass and apparent glucose disposal. $\mathrm{Br} J \mathrm{Nutr}$ 104:407-417.
Niba LL (2003). Processing effects on susceptibility of starch to digestion in some dietary starch sources. Int J Food Sci Nutr 54:97-109.

Panlasigui LN \& Thompson LU (2006). Blood glucose lowering effects of brown rice in normal and diabetic subjects. Int $J$ Food Sci Nutr 57:151-158.

Ranawana D, Henry C, Lightowler H \& Wang D (2009). Glycaemic index of some commercially available rice and rice products in Great Britain. Int J Food Sci Nutr 60:99-110.

Rashmi S \& Urooj A (2003). Effect of processing on nutritionally important starch fractions in rice varieties. Int J Food Sci Nutr 54:27-36.

Reed MO, Ai Y, Leutcher JL \& Jane JL (2013). Effects of cooking methods and starch structures on starch hydrolysis rates of rice. $J$ Food Sci 78:H1076-H1081.

Sánchez-Pardo ME, Ortiz-Moreno A, MoraEscobedo $\mathrm{R}$ \& Necoechea-Mondragón $\mathrm{H}$ (2007). In vitro starch digestibility and predicted glycemic index of microwaved and conventionally baked pound cake. Plant Foods Hum Nutr 62:99-105.

Tian S, Nakamura K \& Kayahara H (2004). Analysis of phenolic compounds in white rice, brown rice, and germinated brown rice. $J$ Agr Food Chem 52:4808-4813.

Toutounji MR, Farahnaky A, Santhakumar AB, Oli P, Butardo VM \& Blanchard CL (2019). Intrinsic and extrinsic factors affecting rice starch digestibility. Trends Food Sci Technol 88:10-22.

Yiu S, Weisz J \& Wood P (1991). Comparison of the effect of microwave and conventional cooking on starch and b-glucan in rolledoats. Cereal Chem 68:372-375.

Zheng Y, Ley SH \& Hu FB (2018). Global aetiology and epidemiology of type 2 diabetes mellitus and its complications. Nat Rev Endocrinol $14: 88$ 